\title{
CEBPA-targeting saRNA MTL-CEBPA Liposome
}

National Cancer Institute

\section{Source}

National Cancer Institute. CEBPA-targeting saRNA MTL-CEBPA Liposome. NCI

Thesaurus. Code C126800.

A lipid-based nanoparticle formulation composed of liposomes encapsulating a small oligonucleotide encoding a small activating RNA (saRNA) targeting the CCAAT enhancer binding protein alpha (CEBPA; C/EBP-a) gene, with potential antineoplastic activity. Although the exact mechanism of action through which saRNAs exert their effect(s) is still largely being investigated, it appears that, upon administration, the CEBPA-targeting saRNA MT L-CEBPA liposome targets and binds to a specific DNA regulatory target region, most likely the promoter region, for the CEBPA gene. This restores CEBPA gene transcription, and increases both CEBPA mRNA levels and protein expression. This in turn activates the expression of tumor suppressor genes and may halt proliferation of susceptible tumor cells. Specifically, upregulation of CEBPA in liver cells abrogates liver cancer cell proliferation, thereby prevents liver failure and normalizes liver function. CEBPA, a transcription factor that plays a key role in the regulation of the expression of genes with many functions, including those involved in cellular proliferation, metastasis and normal hepatocyte function, is found in many tissues, including liver cells, adipose tissue and myeloid cells. CEBPA is downregulated in certain types of cancer cells, such as liver cancer cells. saRNA is a short, double-stranded RNA that is structurally related to siRNAs; saRNA is most likely to bind to a target site on the promoter of the CEBPA gene and upregulates its gene expression. 